\title{
Relations between fractional models with three-parameter Mittag-Leffler kernels
}

\author{
Arran Fernandez ${ }^{1}$, Thabet Abdeljawad ${ }^{2,3,4^{*}}$ and Dumitru Baleanu $u^{5,6}$
}

${ }^{\text {"Correspondence: }}$
tabdeljawad@psu.edu.sa
${ }^{2}$ Department of Mathematics and
General Sciences, Prince Sultan
University, Riyadh, Saudi Arabia
${ }^{3}$ Department of Medical Research,
China Medical University, Taichung,
Taiwan
Full list of author information is
available at the end of the article

available at the end of the article

\begin{abstract}
We consider two models of fractional calculus which are defined using three-parameter Mittag-Leffler functions: the Prabhakar definition and a recently defined extension of the Atangana-Baleanu definition. By examining the relationships between the two, we are able to find some new properties of both, as well as of the original Atangana-Baleanu model and its iterated form.
\end{abstract}

Keywords: Fractional calculus; Mittag-Leffler functions; Atangana-Baleanu fractional calculus; Prabhakar fractional calculus

\section{Introduction}

Fractional calculus is based on a very old idea which has only recently become popular in applications. The idea is to generalise differentiation and integration to non-integer orders in order to extend the theory of calculus and to describe a wider range of possible behaviours in reality.

The most frequent definition of fractional integrals and derivatives is the RiemannLiouville one, in which fractional integrals are defined by

$$
{ }_{a}^{\mathrm{RL}} I_{x}^{\alpha} \phi(x)=\frac{1}{\Gamma(\alpha)} \int_{a}^{x}(x-t)^{\alpha-1} f(t) \mathrm{d} t, \quad \operatorname{Re}(\alpha)>0,
$$

and fractional derivatives are then defined by

$$
{ }_{a}^{\mathrm{RL}} D_{x}^{\alpha} \phi(x)=\frac{\mathrm{d}^{n}}{\mathrm{~d} x^{n}}\left({ }_{a}^{\mathrm{RL}} I_{x}^{n-\alpha} \phi(x)\right), \quad n:=\lfloor\operatorname{Re}\rfloor+1, \operatorname{Re}(\alpha) \geq 0 .
$$

These formulae and their fundamental properties are explored in more depth in books such as $[20,21]$.

Fractional calculus has many connections with special functions, and a major application of fractional calculus within pure mathematics is to prove new relations and identities between special functions. One function with particularly strong ties to fractional calculus is the Mittag-Leffler function [16, 19, 22], defined in the classical case as

$$
E_{\alpha}(z)=\sum_{j=0}^{\infty} \frac{z^{j}}{\Gamma(\alpha j+1)}, \quad \operatorname{Re}(\alpha)>0 .
$$

(c) The Author(s) 2020. This article is licensed under a Creative Commons Attribution 4.0 International License, which permits use, sharing, adaptation, distribution and reproduction in any medium or format, as long as you give appropriate credit to the original author(s) and the source, provide a link to the Creative Commons licence, and indicate if changes were made. The images or other third party material in this article are included in the article's Creative Commons licence, unless indicated otherwise in a credit line to the material. If material is not included in the article's Creative Commons licence and your intended use is not permitted by statutory regulation or exceeds the permitted use, you will need to obtain permission directly from the copyright holder. To view a copy of this licence, visit http://creativecommons.org/licenses/by/4.0/. 
The three-parameter version of this function introduced by Prabhakar [23] is defined as follows:

$$
E_{\alpha, \beta}^{\gamma}(z)=\sum_{j=0}^{\infty} \frac{\Gamma(\gamma+j) z^{j}}{\Gamma(j) j ! \Gamma(\alpha j+\beta)}, \quad \operatorname{Re}(\alpha)>0, \operatorname{Re}(\beta)>0 .
$$

These functions, along with assorted related functions and generalisations, are studied in depth in [15].

It is important to be aware that the Riemann-Liouville formulae are far from being the only way to define fractional derivatives and integrals. There are dozens of different definitions, each of them with different properties and ability to model different behaviours. It has been proposed to group these different models into broad classes, each of which has some particular patterns of behaviour [9]. In general, for pure mathematical studies, it is best to examine these broad classes in order to gain results with the largest possible generality, and then apply the results in specific types of fractional calculus for real-world applications. But here, we wish to examine a few specific definitions in order to understand the relationships between them and whether it is appropriate to consider them separately or not.

In 2016, Atangana and Baleanu [7] proposed a definition of fractional calculus, which replaces the power-function kernel of the Riemann-Liouville definition with a MittagLeffler function kernel. The formulae are as follows for fractional derivatives and integrals respectively:

$$
\begin{aligned}
& { }_{a}^{\mathrm{ABR}} D_{x}^{\alpha} \phi(x)=\frac{B(\alpha)}{1-\alpha} \frac{\mathrm{d}}{\mathrm{d} x} \int_{a}^{x} E_{\alpha}\left(\frac{-\alpha}{1-\alpha}(x-\xi)^{\alpha}\right) \phi(\xi) \mathrm{d} \xi, \\
& { }_{a}^{\mathrm{ABC}} D_{x}^{\alpha} \phi(x)=\frac{B(\alpha)}{1-\alpha} \int_{a}^{x} E_{\alpha}\left(\frac{-\alpha}{1-\alpha}(x-\xi)^{\alpha}\right) \phi^{\prime}(\xi) \mathrm{d} \xi, \\
& { }_{a}^{\mathrm{AB}} I_{x}^{\alpha} \phi(x)=\frac{1-\alpha}{B(\alpha)} \phi(x)+\frac{\alpha}{B(\alpha)}{ }^{\mathrm{RL}} I_{x}^{\alpha} \phi(x),
\end{aligned}
$$

where $x \in(a, b)$ and $\alpha \in(0,1)$ (or complex $\alpha$ as defined in [11]) and $B(\alpha)$ is a multiplier that satisfies $B(0)=B(1)=1$ and that we may assume [8] is in $\mathbb{R}_{0}^{+}$. Here "ABR" and "ABC" denote the $A B$ fractional derivatives of Riemann-Liouville type and Caputo type respectively. The $\mathrm{AB}$ fractional calculus has become popular in recent years largely due to its extensive applications $[6,10,18,24]$.

Recently $[1,4]$ a definition was proposed, inspired by the Atangana-Baleanu definition for fractional integrals and derivatives of $A B$ type with a three-parameter Mittag-Leffler function kernel. In this model, the fractional derivative is given by

$$
\begin{aligned}
& { }_{a}^{\mathrm{ABR}} D_{x}^{\alpha, \mu, \gamma} \phi(x)=\frac{B(\alpha)}{1-\alpha} \frac{\mathrm{d}}{\mathrm{d} x} \int_{a}^{x}(x-\xi)^{\mu-1} E_{\alpha, \mu}^{\gamma}\left(\frac{-\alpha}{1-\alpha}(x-\xi)^{\alpha}\right) \phi(\xi) \mathrm{d} \xi \\
& { }_{a}^{\mathrm{ABC}} D_{x}^{\alpha, \mu, \gamma} \phi(x)=\frac{B(\alpha)}{1-\alpha} \int_{a}^{x}(x-\xi)^{\mu-1} E_{\alpha, \mu}^{\gamma}\left(\frac{-\alpha}{1-\alpha}(x-\xi)^{\alpha}\right) \phi^{\prime}(\xi) \mathrm{d} \xi
\end{aligned}
$$


where $x \in(a, b)$ and $\alpha \in(0,1)$ and $\mu, \gamma \in \mathbb{C}$ with $\operatorname{Re}(\mu)>0$, and the multiplier $B(\alpha)$ is as above, while the fractional integral is given by

$$
{ }_{a}^{\mathrm{AB}} I_{x}^{\alpha, \mu, \gamma} \phi(x)=\sum_{j=0}^{\infty}\left(\begin{array}{l}
\gamma \\
j
\end{array}\right) \frac{\alpha^{j}}{B(\alpha)(1-\alpha)^{j-1}}{ }^{\mathrm{RL}} I_{x}^{j \alpha+1-\mu} \phi(x),
$$

where $x \in(a, b)$ and $\alpha \in(0,1)$ and $\mu, \gamma \in \mathbb{C}$ with $\operatorname{Re}(\mu)<1$. (In the original paper [1], the set of possible values for $\gamma$ is variously stated to be $\mathbb{R}$ or $\mathbb{R}^{+}$or $\mathbb{C}$, but we clarify here that the above formulae are valid for all $\gamma \in \mathbb{C}$.) Discrete versions of these operators were also proposed in [2], but in the current work we shall focus only on the continuous case.

Meanwhile, the AB definition has often been compared with the Prabhakar definition of fractional calculus, which dates back much further [17, 23] and which also involves a three-parameter Mittag-Leffler function kernel. In this model, the fractional integral is given by

$$
{ }_{a}^{P} I_{x}^{\alpha, \beta, \gamma, \delta} \phi(x)=\int_{a}^{x}(x-\xi)^{\beta-1} E_{\alpha, \beta}^{\gamma}\left(\delta(x-\xi)^{\alpha}\right) \phi(\xi) \mathrm{d} \xi
$$

where $x \in(a, b)$ and $\alpha, \beta, \gamma, \delta \in \mathbb{C}$ with $\operatorname{Re}(\alpha)>0$ and $\operatorname{Re}(\beta)>0$, and the fractional derivatives (again of both Riemann-Liouville and Caputo type [14]) are given by

$$
\begin{array}{ll}
{ }_{a}^{\mathrm{PR}} D_{x}^{\alpha, \beta, \gamma, \delta} \phi(x)=\frac{\mathrm{d}^{k}}{\mathrm{~d} x^{k}}{ }^{P} I_{x}^{\alpha, k-\beta,-\gamma, \delta} \phi(x), & k=\lfloor\operatorname{Re}(\beta)\rfloor+1 ; \\
{ }_{a}^{\mathrm{PC}} D_{x}^{\alpha, \beta, \gamma, \delta} \phi(x)={ }_{a}^{P} I_{x}^{\alpha, k-\beta,-\gamma, \delta} \frac{\mathrm{d}^{k}}{\mathrm{~d} x^{k}} \phi(x), & k=\lfloor\operatorname{Re}(\beta)\rfloor+1,
\end{array}
$$

where $x \in(a, b)$ and $\alpha, \beta, \gamma, \delta \in \mathbb{C}$ with $\operatorname{Re}(\alpha)>0$ and $\operatorname{Re}(\beta) \geq 0$. In the first formula (8), $k$ may be replaced by any natural number $n>\lfloor\operatorname{Re}(\beta)\rfloor$ without altering the definition.

All of the above formulae are left-sided, integrals going from $a$ to $x$ in each case. The corresponding right-sided formulae, with integrals going from $x$ to $b$, exist for all the definitions considered here, and their properties are analogous. For simplicity, in the current work we consider only the left-sided versions of each definition, knowing that all the results proved here have analogues for the right-sided versions.

Given the existence of the well-known Prabhakar fractional calculus using a threeparameter Mittag-Leffler function kernel, which has sometimes been called a threeparameter analogue of the $A B$ fractional calculus, the recent introduction of the "threeparameter $A B$ " fractional calculus begs the question of the relationship between the two. Do formulae (4)-(6) and formulae (7)-(9) have any connection, or are they two different models of fractional calculus both using three-parameter Mittag-Leffler functions?

In this paper we demonstrate the connection between these two definitions of fractional calculus and use it to discuss some further properties of both. The main original contributions are Theorems 2.1, 2.2, 3.1, 3.2. Section 2 establishes the direct relationship between Prabhakar and three-parameter $\mathrm{AB}$, with detailed discussion around the restrictions on and relationships between the various parameters involved. The main result here is that the three-parameter AB model is essentially identical to the well-known Prabhakar model, and therefore it is unnecessary to study it separately. Section 3 establishes both the original AB integral and its iterated version as special cases of the Prabhakar model. Although 
the $\mathrm{AB}$ derivative is well known as a case of Prabhakar, our original contribution is to show the AB integral as such. The main result here is that the whole AB model, and even the iterated version that was created for its semigroup property, is contained within the Prabhakar class. This gives a new way of understanding the AB integral: it is not only a weighted sum of a function with its Riemann-Liouville integral, but also a transform with a type of Mittag-Leffler kernel. Section 4 is for the conclusions.

\section{Relating three-parameter AB with Prabhakar}

Theorem 2.1 The relationship between the three-parameter AB integral and the Prabhakar integral is given as follows:

$$
{ }_{a}^{\mathrm{AB}} I_{x}^{\alpha, \mu, \gamma} \phi(x)=\frac{1-\alpha}{B(\alpha)} \cdot{ }_{a}^{P} I_{x}^{\alpha, 1-\mu,-\gamma, \frac{-\alpha}{1-\alpha}} \phi(x)
$$

where $x \in(a, b)$ and the parameters $\alpha, \mu, \gamma \in \mathbb{C}$ satisfy $0<\alpha<1, \operatorname{Re}(\mu)<1$.

Proof We use the following series formula for Prabhakar integrals, which was established in [13]:

$$
{ }_{a}^{P} I_{x}^{\alpha, \beta, \gamma, \delta} \phi(x)=\sum_{j=0}^{\infty} \frac{\Gamma(\gamma+j) \delta^{j}}{\Gamma(\gamma) j !}{ }_{a}^{\mathrm{RL}} I_{x}^{\alpha j+\beta} \phi(x)
$$

and then proceed as follows, using the general identity between gamma functions $\frac{\Gamma(z+n)}{\Gamma(z)}=$ $(-1)^{n} \frac{\Gamma(1-z)}{\Gamma(1-z-n)}$, which follows from the reflection formula.

$$
\begin{aligned}
{ }_{a}^{\mathrm{AB}} I_{x}^{\alpha, \mu, \gamma} \phi(x) & =\sum_{j=0}^{\infty}\left(\begin{array}{l}
\gamma \\
j
\end{array}\right) \frac{\alpha^{j}}{B(\alpha)(1-\alpha)^{j-1} a}{ }^{\mathrm{RL}} I_{x}^{j \alpha+1-\mu} \phi(x) \\
& =\sum_{j=0}^{\infty} \frac{\Gamma(\gamma+1)}{\Gamma(\gamma-j+1) j !}\left(\frac{\alpha}{1-\alpha}\right)^{j}\left(\frac{1-\alpha}{B(\alpha)}\right){ }_{a}^{\mathrm{RL}} I_{x}^{j \alpha+1-\mu} \phi(x) \\
& =\frac{1-\alpha}{B(\alpha)} \sum_{j=0}^{\infty}(-1)^{j} \frac{\Gamma(-\gamma+j)}{\Gamma(-\gamma) j !}\left(\frac{\alpha}{1-\alpha}\right)_{a}^{j}{ }_{a}^{\mathrm{RL}} I_{x}^{j \alpha+1-\mu} \phi(x) \\
& =\frac{1-\alpha}{B(\alpha)} \sum_{j=0}^{\infty} \frac{\Gamma(-\gamma+j)\left(\frac{-\alpha}{1-\alpha}\right)^{j}}{\Gamma(-\gamma) j !}{ }_{a} I_{x}^{j \alpha+1-\mu} \phi(x) \\
& =\frac{1-\alpha}{B(\alpha)} \cdot{ }_{a}^{P} I_{x}^{\alpha, 1-\mu,-\gamma, \frac{-\alpha}{1-\alpha}} \phi(x) .
\end{aligned}
$$

Theorem 2.2 The relationship between the three-parameter AB derivatives and the Prabhakar derivatives is given in general by

$$
\begin{aligned}
& { }_{a}^{\mathrm{ABR}} D_{x}^{\alpha, \mu, \gamma} \phi(x)=\frac{B(\alpha)}{1-\alpha} \cdot \frac{\mathrm{d}}{\mathrm{d} x}{ }_{a}^{P} I_{x}^{\alpha, \mu, \gamma, \frac{-\alpha}{1-\alpha}} \phi(x) ; \\
& { }_{a}^{\mathrm{ABC}} D_{x}^{\alpha, \mu, \gamma} \phi(x)=\frac{B(\alpha)}{1-\alpha} \cdot{ }_{a}^{P} I_{x}^{\alpha, \mu, \gamma}, \frac{-\alpha}{1-\alpha} \phi^{\prime}(x),
\end{aligned}
$$


where $x \in(a, b)$ and the parameters $\alpha, \mu, \gamma \in \mathbb{C}$ satisfy $0<\alpha<1, \operatorname{Re}(\mu)>0$. In the specific case where $0<\operatorname{Re}(\mu)<1$, the relationship is as follows:

$$
\begin{aligned}
& { }_{a}^{\mathrm{ABR}} D_{x}^{\alpha, \mu, \gamma} \phi(x)=\frac{B(\alpha)}{1-\alpha} \cdot{ }_{a}^{\mathrm{PR}} D_{x}^{\alpha, 1-\mu,-\gamma, \frac{-\alpha}{1-\alpha}} \phi(x) ; \\
& { }_{a}^{\mathrm{ABC}} D_{x}^{\alpha, \mu, \gamma} \phi(x)=\frac{B(\alpha)}{1-\alpha} \cdot{ }_{a}^{\mathrm{PC}} D_{x}^{\alpha, 1-\mu,-\gamma, \frac{-\alpha}{1-\alpha}} \phi(x) .
\end{aligned}
$$

Proof The first identities are immediately clear from definitions (4)-(5) of the threeparameter $A B$ derivatives and definition (7) of the Prabhakar integral. We turn therefore to the case of $0<\operatorname{Re}(\mu)<1$.

From definitions (7)-(9) of Prabhakar derivatives and integrals, we have for $0<\operatorname{Re}(\beta)<1$

$$
\begin{aligned}
& { }_{a}^{\mathrm{PR}} D_{x}^{\alpha, \beta, \gamma, \delta} \phi(x)=\frac{\mathrm{d}}{\mathrm{d} x} \int_{a}^{x}(x-\xi)^{-\beta} E_{\alpha, 1-\beta}^{-\gamma}\left(\delta(x-\xi)^{\alpha}\right) \phi(\xi) \mathrm{d} \xi, \\
& { }_{a}^{\mathrm{PC}} D_{x}^{\alpha, \beta, \gamma, \delta} \phi(x)=\int_{a}^{x}(x-\xi)^{-\beta} E_{\alpha, 1-\beta}^{-\gamma}\left(\delta(x-\xi)^{\alpha}\right) \phi^{\prime}(\xi) \mathrm{d} \xi,
\end{aligned}
$$

and so the results for fractional derivatives follow immediately by comparing these expressions with those in (4)-(5).

Remark 2.1 We comment here on the restrictions on the parameters that were imposed in the statement of Theorems 2.1 and 2.2.

The artificial restriction $0<\alpha<1$ is included in both theorems only because this was part of the definition of the three-parameter $A B$ differintegrals as proposed in [1]. We now see that this restriction is mathematically unnecessary, since all the expressions and identities are equally valid for any $\alpha \in \mathbb{C}$ with $\operatorname{Re}(\alpha)>0$.

The restriction $0<\operatorname{Re}(\mu)<1$ is actually required in order for both sides of (14)-(15) to be well defined. The three-parameter AB definitions (4)-(5) and the Prabhakar definitions (8)-(9) represent two possible extensions of the expressions given by "composition of a Prabhakar fractional integral with a first-order derivative" from the restricted domain $0<\operatorname{Re}(\beta)<1$ or $0<\operatorname{Re}(\mu)<1$ to a broader domain. (This is the reason for our usage of different notations $\beta$ and $\mu$ for the second variable in the three-parameter AB and Prabhakar models respectively: their relationship is given by $\beta+\mu=1$.)

- In line with the original $A B$ derivatives (1)-(2), the three-parameter $A B$ definition treats the first-order derivative as a fixed feature of the operator. This definition extends (14)-(15) to the half-plane domain $\operatorname{Re}(\mu)>0$ or $\operatorname{Re}(\beta)<1$.

- In line with the original Riemann-Liouville and Caputo derivatives, the Prabhakar definition allows the order of the non-fractional derivative to vary according to the size of $\operatorname{Re}(\beta)$. This definition extends (14)-(15) to the half-plane domain $\operatorname{Re}(\mu)<1$ or $\operatorname{Re}(\beta)>0$.

The conclusion of the above theorems is that the three-parameter AB integral is always identical with the Prabhakar integral, while the three-parameter AB derivatives are always related to the Prabhakar integral by a simple $\frac{\mathrm{d}}{\mathrm{d} x}$ operation and are identical with the Prabhakar derivatives in the particular domain of interest. 
Proposition 2.1 The three-parameter $A B$ integrals and derivatives satisfy the following quasi-semigroup properties:

$$
\begin{aligned}
& \underset{a}{\mathrm{AB}} I_{x}^{\alpha, \mu_{1}, \gamma_{1} \mathrm{AB}} \underset{a}{\alpha, \mu_{x}, \gamma_{2}} \phi(x)=\frac{1-\alpha}{B(\alpha)}{ }_{a}^{\mathrm{AB}} I_{x}^{\alpha, \mu_{1}+\mu_{2}-1, \gamma_{1}+\gamma_{2}} \phi(x), \\
& \operatorname{Re}(\alpha)>0, \operatorname{Re}\left(\mu_{i}\right)>0 ; \\
& \underset{a}{\mathrm{ABR}} D_{x}^{\alpha, \mu_{1}, \gamma_{1} \mathrm{AB}}{ }_{a}^{\alpha, \mu_{2}, \gamma_{2}} \phi(x)=\frac{1-\alpha}{B(\alpha)}{ }^{\mathrm{ABR}} D_{x}^{\alpha, 1+\mu_{1}-\mu_{2}, \gamma_{1}-\gamma_{2}} \phi(x), \\
& \operatorname{Re}(\alpha)>0, \operatorname{Re}\left(\mu_{1}\right)>0, \operatorname{Re}\left(\mu_{2}\right)<1 .
\end{aligned}
$$

Proof This is an immediate consequence of the well-known semigroup property of Prabhakar fractional integrals $[17,23]$ :

$$
{ }_{a}^{P} I_{x}^{\alpha, \beta_{1}, \gamma_{1}, \delta P}{ }_{a}^{\alpha, \beta_{2}, \gamma_{2}, \delta} \phi(x)={ }_{a}^{P} I_{x}^{\alpha, \beta_{1}+\beta_{2}, \gamma_{1}+\gamma_{2}, \delta} \phi(x), \quad \operatorname{Re}(\alpha)>0, \operatorname{Re}\left(\beta_{i}\right)>0 .
$$

We note that (18) is a true semigroup property, the parameters $\beta_{1}, \beta_{2}$ and $\gamma_{1}, \gamma_{2}$ on the left-hand side being replaced by $\beta_{1}+\beta_{2}$ and $\gamma_{1}+\gamma_{2}$ on the right-hand side. There is also a natural semigroup property for Prabhakar derivatives and integrals:

$$
\begin{gathered}
{ }_{a}^{\mathrm{PR}} D_{x}^{\alpha, \beta_{1}, \gamma_{1}, \delta P}{ }_{a}^{\alpha, \beta_{2}, \gamma_{2}, \delta} \phi(x)={ }_{a}^{\mathrm{PR}} D_{x}^{\alpha, \beta_{1}-\beta_{2}, \gamma_{1}-\gamma_{2}, \delta} \phi(x), \\
\operatorname{Re}(\alpha)>0, \operatorname{Re}\left(\beta_{1}\right)>\operatorname{Re}\left(\beta_{2}\right)>0 .
\end{gathered}
$$

Remark 2.2 The semigroup identity (16) was already proved in the original paper [1] that proposed the three-parameter $\mathrm{AB}$ operators. We have presented it again here, along with the new identity (17), just to show how easily it follows from the known results on Prabhakar operators.

Comparing formulae (16)-(17) for three-parameter $\mathrm{AB}$ semigroup properties with the corresponding formulae (18)-(19) for Prabhakar semigroup properties, we see that the former are much clunkier. The reason for this is that the mathematically natural semigroup property is in the second and third parameters of the Prabhakar operators, without any multipliers outside the integrals. For the three-parameter $\mathrm{AB}$ operators, we use the second parameter $\mu=1-\beta$ instead of $\beta$, and the multiplier functions $B(\alpha)$ are included; thus, the right-hand sides of (16)-(17) must involve $\mu_{1} \pm \mu_{2} \mp 1$ instead of just $\beta_{1} \pm \beta_{2}$ and must contain an extra $\frac{1-\alpha}{B(\alpha)}$ factor instead of a direct semigroup property.

Corollary 2.1 The three-parameter AB integral and three-parameter $A B R$ derivative have the following inversion property:

$$
{ }_{a}^{\mathrm{ABR}} D_{x}^{\alpha, \mu, \gamma} \underset{a}{\mathrm{AB}} I_{x}^{\alpha, \mu, \gamma} \phi(x)=\phi(x), \quad \operatorname{Re}(\alpha)>0,0<\operatorname{Re}(\mu)<1 .
$$

Proof This follows directly from equation (17) in the previous proposition, noting that the function $E_{\alpha, 1}^{0}$ is identically 1 .

The above corollary is an inversion property in the three-parameter $A B$ model, analogous to the known relation between the original $A B R$ derivative and $A B$ integral. The 
corresponding relationship between the original $A B C$ derivative and $A B$ integral is of a Newton-Leibniz form [3], and it too has an analogue in the three-parameter AB model $[1$, Theorem 3]. We now confirm the following similar result.

Proposition 2.2 The Prabhakar integral and the Caputo-type Prabhakar derivative satisfy the following Newton-Leibniz formula:

$$
{ }_{a}^{P} I_{x}^{\alpha, \beta, \gamma, \delta \mathrm{PC}} D_{x}^{\alpha, \beta, \gamma, \delta} \phi(x)=\phi(x)-\phi(a), \quad \operatorname{Re}(\alpha)>0,0<\operatorname{Re}(\beta)<1 .
$$

Proof This is an immediate consequence of the Newton-Leibniz property in the threeparameter $\mathrm{AB}$ model:

$$
{ }_{a}^{\mathrm{AB}} I_{x}^{\alpha, \mu, \gamma} \underset{a}{\mathrm{ABC}} D_{x}^{\alpha, \mu, \gamma} \phi(x)=\phi(x)-\phi(a), \quad \operatorname{Re}(\alpha)>0,0<\operatorname{Re}(\mu)<1 .
$$

Alternatively, the result is easy to prove from the definition of the Caputo-type Prabhakar derivative and the semigroup property of the Prabhakar integral. Indeed, it is surprising that, as far as we know, the result of this proposition has not been stated explicitly before.

$$
\begin{aligned}
{ }_{a}^{P} I_{x}^{\alpha, \beta, \gamma, \delta P C} D_{x}^{\alpha, \beta, \gamma, \delta} \phi(x) & ={ }_{a}^{P} I_{x}^{\alpha, \beta, \gamma, \delta P}{ }_{a}^{\alpha, 1-\beta, \gamma, \delta} \frac{\mathrm{d}}{\mathrm{d} x} \phi(x) \\
& ={ }_{a}^{P} I_{x}^{\alpha, 1,0, \delta} \phi^{\prime}(x) \\
& =\int_{a}^{x} \phi^{\prime}(\xi) \mathrm{d} \xi=\phi(x)-\phi(a) .
\end{aligned}
$$

Remark 2.3 Note that the condition $0<\operatorname{Re}(\beta)<1$ or $0<\operatorname{Re}(\mu)<1$ is necessary for both (21) and (22). In [1, Theorem 3] the condition was stated as $\mu>0$, but the results are not valid for all $\mu>0$ : indeed, the three-parameter $\mathrm{AB}$ integral (6) is only defined for $\operatorname{Re}(\mu)<1$.

We also note in passing that, in [1, Equation (36)], the correct right-hand side is $\theta(b)-$ $\theta(t)$ and not $\theta(t)-\theta(b)$.

In the following proposition, we correct an error which appeared in [1, Theorem 6]. In that theorem, the results were divided into two cases $(\mu \neq 1$ and $\mu=1)$, but in fact the equation for $\mu \neq 1$ contains an error, and the argument used is only valid when $\operatorname{Re}(\mu)>1$. We state the correct result as follows.

Proposition 2.3 The three-parameter ABR derivative satisfies the following series formula:

$$
\begin{aligned}
& { }_{a}^{\mathrm{ABR}} D_{x}^{\alpha, \mu, \gamma} \phi(x)=\frac{B(\alpha)}{1-\alpha} \sum_{j=0}^{\infty}\left(\frac{-\alpha}{1-\alpha}\right)^{j} \frac{\Gamma(\gamma+j)}{\Gamma(\gamma) j !}{ }_{a}^{\mathrm{RL}} I_{x}^{\alpha j+\mu-1} \phi(x), \\
& \alpha, \mu, \gamma \in \mathbb{C}, \operatorname{Re}(\alpha), \operatorname{Re}(\mu)>0 .
\end{aligned}
$$

Proof The argument used in the proof of [1, Theorem 6] is correct except for the claim that $\left[z^{\mu-1} E_{\alpha, \mu}^{\gamma}\left(\lambda z^{\alpha}\right)\right]_{z=0}=\frac{1}{\Gamma(\mu)}$. In fact, we have from the definition

$$
z^{\mu-1} E_{\alpha, \mu}^{\gamma}\left(\lambda z^{\alpha}\right)=\frac{z^{\mu-1}}{\Gamma(\mu)}+\frac{\lambda \Gamma(\gamma+1) z^{\alpha+\mu-1}}{1 ! \Gamma(\gamma) \Gamma(\alpha+\mu)}+\frac{\lambda^{2} \Gamma(\gamma+2) z^{2 \alpha+\mu-1}}{2 ! \Gamma(\gamma) \Gamma(2 \alpha+\mu)}+\cdots
$$


In the case $\operatorname{Re}(\alpha)>0$ and $\operatorname{Re}(\mu)>1$, this function is equal to zero at $z=0$. In the case $\operatorname{Re}(\alpha)>0$ and $0<\operatorname{Re}(\mu)<1$, it goes to infinity as $z \rightarrow 0$. It is only equal to $\frac{1}{\Gamma(\mu)}$ at $z=0$ in the special case $\mu=1$.

Therefore, for $\operatorname{Re}(\mu)>1$, the argument given in [1, Theorem 6] would be correct with the extra first term outside the series removed. For $0<\operatorname{Re}(\mu) \leq 1$, this method of proof is no longer valid, since the boundary term from the integral would be infinite. We note that the identity

$$
\frac{\mathrm{d}}{\mathrm{d} x} \int_{a}^{x} G(x, \xi) \mathrm{d} \xi=G(x, x)+\int_{a}^{x} \frac{\partial}{\partial x} G(x, \xi) \mathrm{d} \xi
$$

is only valid if the integral on the left-hand side is non-singular, i.e. if $\lim _{\xi \rightarrow x} G(x, \xi)$ exists. In our case, the integral from definition (4) of three-parameter ABR derivatives is singular for $0<\operatorname{Re}(\mu)<1$ and non-singular for $\operatorname{Re}(\mu)>1$.

Although the method of proof from [1, Theorem 6] no longer works when $0<\operatorname{Re}(\mu) \leq 1$, the result is still true in this case. In fact, using the known series formula (11) for Prabhakar integrals, together with connection (12) that we proved in Theorem 2.2, gives the desired result immediately for all values of $\mu$, under the assumptions $\operatorname{Re}(\alpha), \operatorname{Re}(\mu)>0$, which are included in the definition of the three-parameter ABR derivative.

Remark 2.4 In connection with Proposition 2.3, it is of interest to compare with the discrete case which is given in [2, Theorem 6]. The discrete result is

$$
\begin{aligned}
& { }_{a}^{\mathrm{ABR}} \nabla_{x}^{\alpha, \mu, \gamma} \phi(x)=\frac{B(\alpha)}{1-\alpha} \sum_{j=0}^{\infty}\left(\frac{-\alpha}{1-\alpha}\right)^{j} \frac{\Gamma(\gamma+j)}{\Gamma(\gamma) j !}{ }_{a}^{\mathrm{RL}} \nabla_{x}^{-\alpha j-\mu+1} \phi(x), \\
& \alpha \in\left(0, \frac{1}{2}\right), \mu, \gamma \in \mathbb{C}
\end{aligned}
$$

which we note is exactly analogous (as might be expected) to the correct result (23) in the continuous case.

Remark 2.5 After correcting [1, Theorem 6] by our Proposition 2.3, we rewrite [1, Remark 5, part (3)] as follows.

By Proposition 2.3 above with $\gamma=1$, we notice that

$$
{ }_{a}^{\mathrm{ABR}} D_{x}^{\alpha, \mu, 1} \phi(x)=\frac{B(\alpha)}{1-\alpha} \sum_{j=0}^{\infty}\left(\frac{-\alpha}{1-\alpha}\right)_{a}^{j} I_{x}^{\mathrm{RL}} I^{\alpha j+\mu-1} \phi(x),
$$

which is different from the expression ${ }_{a}^{\mathrm{AB}} I_{x}^{\alpha, \mu, 1,-1} \phi(x)$ defined in [1, Definition 6]. In fact, we have

$$
{ }_{a}^{\mathrm{ABR}} D_{x}^{\alpha, 2-\mu, 1} \phi(t)={ }_{a}^{\mathrm{AB}} I_{x}^{\alpha, \mu, 1,-1} \phi(x) .
$$

This is the same as [1, Eq. (71)] without the incorrect extra term. In the case of $\mu=1$, the result of $[1$, Remark 5, part (3)] is correct. 


\section{Consequences for the original $A B$ and iterated $A B$}

Thus far, we have focused on the relationships between two different models of fractional calculus, Prabhakar and three-parameter AB, which both use integrals with threeparameter Mittag-Leffler function kernels. Although we discovered in the previous section that these models are essentially the same, it is important to remember the initial motivations that inspired them.

The Prabhakar definition has its origin in a paper [23] of 1971, long before fractional calculus with Mittag-Leffler kernels became mainstream. It has been noted in the literature, for example [13], that the AB derivative is a special case of the Prabhakar model-an important special case which is particularly useful in applications-but the AB integral has always been treated as a separate entity, closely related to the Riemann-Liouville integral but never treated as a special case of Prabhakar.

The three-parameter $\mathrm{AB}$ definition, on the other hand, is much more recent and directly inspired by the $A B$ fractional calculus. In the original paper [1], the three-parameter $A B$ derivative was defined by direct analogy with the $A B$ fractional derivative, adding two extra parameters, while the three-parameter AB integral was constructed step-by-step starting from the simple cases of $\gamma=1$ and $\gamma \in \mathbb{N}$.

For this reason, the work of [1] connecting the AB integral with the three-parameter $\mathrm{AB}$ integral, combined with our previous section connecting the three-parameter $\mathrm{AB}$ integral with the Prabhakar integral, may be used to achieve the following interesting results.

Theorem 3.1 The AB fractional integral can be written as a special case of the Prabhakar integral as follows:

$$
{ }_{a}^{\mathrm{AB}} I_{x}^{\alpha} \phi(x)=\frac{1-\alpha}{B(\alpha)} \cdot{ }_{a}^{P} I_{x}^{\alpha, 0,-1, \frac{-\alpha}{1-\alpha}} \phi(x)
$$

for any $\alpha \in \mathbb{C}$.

Proof From [1, Definition 4] it is known that setting $\gamma=1$ in the three-parameter AB integral gives an expression similar to the original $\mathrm{AB}$ integral

$$
{ }_{a}^{\mathrm{AB}} I_{x}^{\alpha, \mu, 1} \phi(x)=\frac{1-\alpha}{B(\alpha)}{ }^{\mathrm{RL}} I_{x}^{1-\mu} \phi(x)+\frac{\alpha}{B(\alpha)}{ }^{\mathrm{RL}} I_{x}^{1-\mu+\alpha} \phi(x),
$$

and in particular, setting $\mu=\gamma=1$ reduces to the AB integral itself

$$
{ }_{a}^{\mathrm{AB}} I_{x}^{\alpha, 1,1} \phi(x)=\frac{1-\alpha}{B(\alpha)} \phi(x)+\frac{\alpha}{B(\alpha)}{ }^{\mathrm{RL}} I_{x}^{\alpha} \phi(x)={ }_{a}^{\mathrm{AB}} I_{x}^{\alpha} \phi(x) .
$$

Combining (25) with the result of Theorem 2.1, we obtain the result. (Note that the original $\mathrm{AB}$ integral was defined only for $0<\alpha<1$, but there is a natural extension to $\alpha \in \mathbb{C}$ as established in [11].)

Alternatively, we can use the series formula (11) and the fact that $(-1)_{j}=0$ for $j \geq 2$ to obtain the result directly:

$$
\frac{1-\alpha}{B(\alpha)}{ }_{a} I_{x}^{\alpha, 0,-1} \phi(x)=\sum_{j=0}^{\infty} \frac{(-1)_{j}}{j !}\left(\frac{-\alpha}{1-\alpha}\right)^{j}{ }_{a}^{R L} I_{x}^{\alpha j+0} \phi(x)
$$




$$
\begin{aligned}
& =\frac{1-\alpha}{B(\alpha)} \cdot \frac{1}{0 !}\left(\frac{-\alpha}{1-\alpha}\right){ }_{a}^{0} I_{x}^{0} \phi(x)+\frac{1-\alpha}{B(\alpha)} \cdot \frac{(-1)}{1 !}\left(\frac{-\alpha}{1-\alpha}\right){ }_{a}^{1} I_{x}^{\alpha} \phi(x) \\
& =\frac{1-\alpha}{B(\alpha)} \phi(x)-\frac{-\alpha}{B(\alpha)}{ }_{a}^{\mathrm{RL}} I_{x}^{\alpha} \phi(x)={ }_{a}^{\mathrm{AB}} I_{x}^{\alpha} \phi(x) .
\end{aligned}
$$

Remark 3.1 It is already known [13] that the AB derivative can be seen as a special case of Prabhakar fractional calculus:

$$
\begin{aligned}
\underset{a}{\mathrm{ABR}} D_{x}^{\alpha} \phi(x) & =\frac{1-\alpha}{B(\alpha)} \cdot \frac{\mathrm{d}}{\mathrm{d} x}{ }_{a}^{P} I_{x}^{\alpha, 1,1, \frac{-\alpha}{1-\alpha}} \phi(x) \\
& =\frac{1-\alpha}{B(\alpha)} \cdot{ }_{a}^{P} D_{x}^{\alpha, 0,-1, \frac{-\alpha}{1-\alpha}} \phi(x) .
\end{aligned}
$$

Combining (26) with our new result (24), we see that AB fractional calculus (with parameter $\alpha$ ) is completely contained within Prabhakar fractional calculus (with parameters $\alpha$, $\beta, \gamma, \delta$ ) when we set

$$
\beta=0, \quad \gamma=-1, \quad \delta=\frac{-\alpha}{1-\alpha} \text {. }
$$

Remark 3.2 It is known that the AB fractional operators have no semigroup property [8], while the Prabhakar fractional operators have a semigroup property in two variables [13, 17]. If $A B$ is a special case of Prabhakar, how is this possible?

In (24) and (26), two of the four Prabhakar parameters are constant. These two, $\beta$ and $\gamma$, are precisely those in which Prabhakar has a semigroup property. This explains why AB has no semigroup property in $\alpha$ : even when seen as a special case of Prabhakar, $\alpha$ is the wrong parameter in which to seek a semigroup property.

It is also clear that, by iterating the $\mathrm{AB}$ integral multiple times and using the Prabhakar semigroup property via (24), the parameter $\beta=0$ will be unchanged, while the parameter $\gamma=-1$ will become minus the order of iteration. This leads us to a definition introduced in [12] (with a discrete version defined in [5]), the so-called iterated $A B$ fractional calculus. It is obtained by taking successive iterations of the $A B$ integral and then fractionalising the result. This model has two parameters, and the fractional integrals and derivatives are defined respectively as follows:

$$
\begin{aligned}
& { }_{a}^{\mathrm{AB}} I_{x}^{\alpha, \kappa} \phi(x)=\sum_{j=0}^{\infty}\left(\begin{array}{c}
\kappa \\
j
\end{array}\right) \frac{(1-\alpha)^{\kappa-j} \alpha^{j}}{B(\alpha)^{\kappa}}{ }_{a}^{\mathrm{LL}} I_{x}^{\alpha j} \phi(x), \\
& { }_{a}^{\mathrm{AB}} D_{x}^{\alpha, \kappa} \phi(x)=\sum_{j=0}^{\infty}\left(\begin{array}{c}
-\kappa \\
j
\end{array}\right) \frac{\alpha^{j} B(\alpha)^{\kappa}}{(1-\alpha)^{\kappa+j}}{ }^{\mathrm{RL}} I_{x}^{\alpha j} \phi(x) .
\end{aligned}
$$

Formulae (27) and (28) are identical except for swapping $\kappa$ with $-\kappa$ : the iterated AB integral and iterated $A B$ derivative are both the same thing with this parameter negated. In general we call them both iterated $A B$ differintegrals. In the original paper [12], it was assumed that $\kappa \in \mathbb{R}$; however, the definitions work for any complex $\kappa$, so we here relax the assumption to $\kappa \in \mathbb{C}$. 
Theorem 3.2 The iterated AB fractional differintegral can be written as a special case of the Prabhakar differintegral as follows:

$$
{ }_{a}^{\mathrm{AB}} I_{x}^{\alpha, \kappa} \phi(x)=\left(\frac{1-\alpha}{B(\alpha)}\right){ }_{a}^{P} I_{x}^{\alpha, 0,-\kappa, \frac{-\alpha}{1-\alpha}} \phi(x)
$$

for any $\alpha, \kappa \in \mathbb{C}$.

Proof For $\kappa=k \in \mathbb{N}$, this follows from the result of the previous theorem and the known semigroup property (18) of Prabhakar integrals:

$$
\begin{aligned}
{ }_{a}^{\mathrm{AB}} I_{x}^{\alpha, k} \phi(x) & =\left({ }_{a}^{\mathrm{AB}} I_{x}^{\alpha}\right)^{k} \phi(x)=\left(\frac{1-\alpha}{B(\alpha)} \cdot{ }_{a}^{P} I_{x}^{\alpha, 0,-1}\right)^{k} \phi(x) \\
& =\left(\frac{1-\alpha}{B(\alpha)}\right){ }_{a}^{P} I_{x}^{\alpha, 0 \times k,-1 \times k} \phi(x)=\left(\frac{1-\alpha}{B(\alpha)}\right){ }_{a}^{P} I_{x}^{\alpha, 0,-k} \phi(x) .
\end{aligned}
$$

In general, we can again use the series formula (11) for Prabhakar integrals:

$$
\begin{aligned}
& \left(\frac{1-\alpha}{B(\alpha)}\right)^{\kappa}{ }_{a} I_{x}^{\alpha, 0,-\kappa, \frac{-\alpha}{1-\alpha}} \phi(x) \\
& =\left(\frac{1-\alpha}{B(\alpha)}\right)^{\kappa} \sum_{j=0}^{\infty} \frac{\Gamma(-\kappa+j)}{\Gamma(-\kappa) j !}\left(\frac{-\alpha}{1-\alpha}\right)_{a}^{j}{ }_{a}^{\mathrm{RL}} I_{x}^{\alpha j+0} \phi(x) \\
& =\sum_{j=0}^{\infty} \frac{(1-\alpha)^{\kappa-j} \alpha^{j}}{B(\alpha)^{\kappa}}(-1)^{j} \frac{\Gamma(-\kappa+j)}{\Gamma(-\kappa) j !}{ }_{a}^{\mathrm{RL}} I_{x}^{\alpha j} \phi(x) \\
& =\sum_{j=0}^{\infty} \frac{(1-\alpha)^{\kappa-j} \alpha^{j}}{B(\alpha)^{\kappa}} \cdot \frac{\Gamma(1+\kappa)}{\Gamma(1+\kappa-j) j !}{ }^{\mathrm{RL}} I_{x}^{\alpha j} \phi(x) \\
& =\sum_{j=0}^{\infty} \frac{(1-\alpha)^{\kappa-j} \alpha^{j}}{B(\alpha)^{\kappa}}\left(\begin{array}{c}
\kappa \\
j
\end{array}\right){ }_{a}^{\mathrm{RL}} I_{x}^{\alpha j} \phi(x) \\
& ={ }_{a}^{\mathrm{AB}} I_{x}^{\alpha, \kappa} \phi(x) .
\end{aligned}
$$

We have now established, inspired by the ideas of [1] and using the series formula from [13], that both the $A B$ integrals and the iterated $A B$ differintegral, as well as the $A B$ derivatives, may be seen as special cases of the Prabhakar fractional calculus.

\section{Conclusions}

In this paper, we have performed a comparative study of two related models of fractional calculus and some of their special cases. We have demonstrated that the recent threeparameter AB formula defines exactly the same model of fractional calculus as the Prabhakar formula. This new connection will be important for researchers working with threeparameter Mittag-Leffler functions in fractional calculus, since now they can work only with the original Prabhakar model.

The original $A B$ fractional calculus of 2016 has become very popular for its applications in various fields of science and engineering. Unlike the Prabhakar fractional calculus, $A B$ has no semigroup property, and the iterated $A B$ fractional calculus was proposed to resolve this lack. Motivated by the three-parameter extension of $A B$, we have now proved 
that the original $\mathrm{AB}$ integral and iterated $\mathrm{AB}$ differintegral are also special cases of Prabhakar. This was already known before for $A B$ derivatives, but now it is seen that the whole $\mathrm{AB}$ fractional calculus is included within the general class of Prabhakar operators. Because that class is closed under composition, this means the iterated $\mathrm{AB}$ model is also included within the Prabhakar class. Of course, the AB model is still a very important special case due to its diverse applications, but it is important to be aware of its status as an element of a larger class of operators [9]. It is also useful to recall that the AB operators are nonsingular even though the Prabhakar operators are in most cases singular: a special case of a typically singular operator may still be non-singular.

We believe these results and interrelationships, this new understanding of fractional calculus with Mittag-Leffler function kernels, will be vital information for the future studies of these models of fractional calculus.

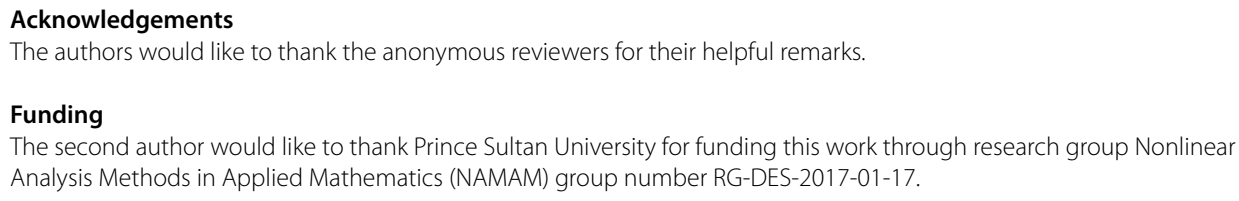

'Department of Mathematics, Faculty of Arts and Sciences, Eastern Mediterranean University, Famagusta, Northern Cyprus, via Mersin 10, Turkey. ${ }^{2}$ Department of Mathematics and General Sciences, Prince Sultan University, Riyadh, Saudi Arabia. ${ }^{3}$ Department of Medical Research, China Medical University, Taichung, Taiwan. ${ }^{4}$ Department of Computer Science and Information Engineering, Asia University, Taichung, Taiwan. ${ }^{5}$ Department of Mathematics, Cankaya University, Ankara, Turkey. ${ }^{6}$ Institute of Space Sciences, Magurele-Bucharest, Romania.

\section{Publisher's Note}

Springer Nature remains neutral with regard to jurisdictional claims in published maps and institutional affiliations.

Received: 6 November 2019 Accepted: 14 April 2020 Published online: 28 April 2020

\section{References}

1. Abdeljawad, T.: Fractional operators with generalized Mittag-Leffler kernels and their iterated differintegrals. Chaos 29, 023102 (2019). https://doi.org/10.1063/1.5085726

2. Abdeljawad, T.: Fractional difference operators with discrete generalized Mittag-Leffler kernels. Chaos Solitons Fractals 126, 315-324 (2019)

3. Abdeljawad, T., Baleanu, D.: Integration by parts and its applications of a new nonlocal fractional derivative with Mittag-Leffler nonsingular kernel. J. Nonlinear Sci. Appl. 10(3), 1098-1107 (2017)

4. Abdeljawad, T., Baleanu, D.: On fractional derivatives with generalized Mittag-Leffler kernels. Adv. Differ. Equ. 2018, 468 (2018). https://doi.org/10.1186/s13662-018-1914-2

5. Abdeljawad, T., Fernandez, A.: On a new class of fractional difference-sum operators with discrete Mittag-Leffler kernels. Mathematics 7(9), 772 (2019). https://doi.org/10.3390/math7090772

6. Acay, B., Bas, E., Abdeljawad, T.: Fractional economic models based on market equilibrium in the frame of different type kernels. Chaos Solitons Fractals 130, 109438 (2020)

7. Atangana, A., Baleanu, D.: New fractional derivative with non-local and non-singular kernel. Therm. Sci. 20(2), 757-763 (2016)

8. Baleanu, D., Fernandez, A.: On some new properties of fractional derivatives with Mittag-Leffler kernel. Commun. Nonlinear Sci. Numer. Simul. 59, 444-462 (2018)

9. Baleanu, D., Fernandez, A.: On fractional operators and their classifications. Mathematics 7(9), 830 (2019). https://doi.org/10.3390/math7090830

10. Baleanu, D., Jajarmi, A., Sajjadi, S.S., Mozyrska, D.: A new fractional model and optimal control of a tumor-immune surveillance with non-singular derivative operator. Chaos 29, 083127 (2019). https://doi.org/10.1063/1.5096159 
11. Fernandez, A:: A complex analysis approach to Atangana-Baleanu fractional calculus. Math. Methods Appl. Sci. (2019). https://doi.org/10.1002/mma.5754

12. Fernandez, A., Baleanu, D.: On a new definition of fractional differintegrals with Mittag-Leffler kernel. Filomat 33(1), 245-254 (2019)

13. Fernandez, A., Baleanu, D., Srivastava, H.M.: Series representations for models of fractional calculus involving generalised Mittag-Leffler functions. Commun. Nonlinear Sci. Numer. Simul. 67, 517-527 (2019)

14. Garra, R., Gorenflo, R., Polito, F., Tomovski, Ž.: Hilfer-Prabhakar derivatives and some applications. Appl. Math. Comput. 242, 576-589 (2014)

15. Gorenflo, R., Kilbas, A.A., Mainardi, F., Rogosin, S.V.: Mittag-Leffler Functions, Related Topics and Applications. Springer, Berlin (2016)

16. Haubold, H.J., Mathai, A.M., Saxena, R.K.: Mittag-Leffler functions and their applications. J. Appl. Math. 2011, 298628 (2011). https://doi.org/10.1155/2011/298628

17. Kilbas, A.A., Saigo, M., Saxena, R.K.: Generalized Mittag-Leffler function and generalized fractional calculus operators, Integral Transforms Spec. Funct. 15(1), 31-49 (2004)

18. Kumar, D., Singh, J., Tanwar, K., Baleanu, D.: A new fractional exothermic reactions model having constant heat source in porous media with power, exponential and Mittag-Leffler laws. Int. J. Heat Mass Transf. 138, 1222-1227 (2019)

19. Mathai, A.M., Haubold, H.J.: Mittag-Leffler functions and fractional calculus. In: Mathai, A.M., Haubold, H.J. (eds.) Special Functions for Applied Scientists, pp. 79-134. Springer, Berlin (2008)

20. Miller, K.S., Ross, B.: An Introduction to the Fractional Calculus and Fractional Differential Equations. Wiley, New York (1993)

21. Oldham, K.B., Spanier, J.: The Fractional Calculus. Academic Press, San Diego (1974)

22. Özarslan, M.A., Kürt, C.: Bivariate Mittag-Leffler functions arising in the solutions of convolution integral equation with 2D-Laguerre-Konhauser polynomials in the kernel. Appl. Math. Comput. 347, 631-644 (2019)

23. Prabhakar, T.R.: A singular integral equation with a generalized Mittag Leffler function in the kernel. Yokohama Math. J. 19, 7-15 (1971)

24. Uçar, S., Uçar, E., Özdemir, N., Hammouch, Z.: Mathematical analysis and numerical simulation for a smoking model with Atangana-Baleanu derivative. Chaos Solitons Fractals 118, 300-306 (2019)

\section{Submit your manuscript to a SpringerOpen ${ }^{\circ}$ journal and benefit from:}

- Convenient online submission

- Rigorous peer review

- Open access: articles freely available online

- High visibility within the field

- Retaining the copyright to your article

Submit your next manuscript at $\boldsymbol{\nabla}$ springeropen.com 